\title{
A Multicenter Clinical Trial on the Diagnostic Value of Dual-Tracer PET/CT in Pulmonary Lesions Using 3'-Deoxy-3'-18 F-Fluorothymidine and ${ }^{18} \mathrm{~F}-\mathrm{FDG}$
}

Jiahe Tian ${ }^{1}$, Xiaofeng Yang ${ }^{2}$, Lijuan $\mathrm{Yu}^{3}$, Ping $\mathrm{Chen}^{4}$, Jun Xin ${ }^{5}$, Liming $\mathrm{Ma}^{6}$, Huiru Feng ${ }^{7}$, Yieyin Tan ${ }^{1}$, Zhoushe $\mathrm{Zhao}^{8}$, and Wenkai $\mathrm{Wu}^{9}$

${ }^{I}$ Department of Nuclear Medicine, The Chinese PLA General Hospital, Beijing, China; ${ }^{2}$ Department of Nuclear Medicine, The People's Hospital of Xinjiang Uygur Autonomous Region, Urumuqi, China; ${ }^{3}$ Department of Medical Imaging, The Tumor Hospital Affiliated to Harbin Medical University, Heilongjiang, China; ${ }^{4}$ PET/CT Center, The 1st Affiliated Hospital of Guangzhou Medical University, Guanzhou, China; ${ }^{5}$ Department of Radiology, The 2nd Affiliated Hospital of China Medical University, Shenyang, China; ${ }^{6}$ Department of Nuclear Medicine, The Kunming General Hospital, Yunan, China; ${ }^{7}$ Department of Nuclear Medicine, The General Hospital of Beijing Command, Beijing, China; ${ }^{8}$ Health Care System, GE China, Beijing, China; and ${ }^{9}$ Department of Nuclear Medicine, Cancer Hospital, Peking Union Medical College, Chinese Academy of Medical Sciences, Beijing, China

Some new radiotracers might add useful information and improve diagnostic confidence of ${ }^{18} \mathrm{~F}-\mathrm{FDG}$ imaging in tumors. A multicenter clinical trial was designed to investigate the diagnostic performance of dual-tracer $\left({ }^{18} \mathrm{~F}-\mathrm{FDG}\right.$ and $3^{\prime}$-deoxy$3^{\prime}-{ }^{18} \mathrm{~F}$-fluorothymidine [18 F-FLT]) PET/CT in pulmonary nodules. Methods: Fifty-five patients underwent dual-tracer imaging in 6 imaging centers using the same models of equipment and standardized protocols. The images were interpreted by a collective group of readers who were unaware of the clinical data. The diagnostic performance using either tracer alone or dual-tracers together, with or without CT, was compared. The histological diagnosis or clinical findings in a 12-mo follow-up period served as the standard of truth. Results: In 16 patients with malignant tumor, 16 with tuberculosis, and 23 with other benign lesions, the sensitivity and specificity of ${ }^{18} \mathrm{~F}-\mathrm{FDG}$ and ${ }^{18} \mathrm{~F}-\mathrm{FLT}$ were $87.5 \%$ and $58.97 \%$ and $68.75 \%$ and $76.92 \%$, respectively. The combination of dual-tracer PET/CT improved the sensitivity and specificity up to $100 \%$ and $89.74 \%$. The 3 subgroups of patients could be best separated when the ${ }^{18} \mathrm{~F}-\mathrm{FLT} /{ }^{18} \mathrm{~F}-\mathrm{FDG}$ standardized uptake value ratio of $0.4-0.90$ was used as the threshold. Conclusion: By reflecting different biologic features, the dualtracer PET/CT using ${ }^{18} \mathrm{~F}-\mathrm{FDG}$ and ${ }^{18} \mathrm{~F}-\mathrm{FLT}$ favorably affected the diagnosis of lung nodules.

Key Words: pulmonary nodules; ${ }^{18} \mathrm{~F}-\mathrm{FDG}$; $3^{\prime}$-deoxy-3'-18 Ffluorothymidine; dual-tracer PET/CT; multicenter clinical trial

J Nucl Med 2008; 49:186-194

DOI: 10.2967/jnumed.107.044966

Received Jul. 5, 2007; revision accepted Nov. 12, 2007

For correspondence or reprints contact: Jiahe Tian, MD, Department of Nuclear Medicine, The Chinese PLA General Hospital, Beijing, China, 100853. E-mail: tianjh@vip.sina.com.cn

COPYRIGHT @ 2008 by the Society of Nuclear Medicine, Inc. t has been known for years that ${ }^{18} \mathrm{~F}$-FDG PET is of little value in certain types of tumors in spite of its wide acceptance in clinical oncology. One of the major shortcomings of ${ }^{18} \mathrm{~F}-\mathrm{FDG}$ was its nonspecific uptake by some benign lesions (1). Therefore, several alternative PET tracers were developed and tried to complement ${ }^{18} \mathrm{~F}-\mathrm{FDG}$. Among them $3^{\prime}$-deoxy- $3^{\prime}-{ }^{18} \mathrm{~F}$-fluorothymidine $\left({ }^{18} \mathrm{~F}-\mathrm{FLT}\right)$ is receiving greater interest because it is an analog of thymidine and its uptake reflects cellular proliferation. In vitro and in vivo studies had shown a higher uptake of ${ }^{18} \mathrm{~F}$-FLT by the proliferating tumors $(2,3)$. In a study on non-small cell lung cancer, a correlation was found between ${ }^{18} \mathrm{~F}-\mathrm{FLT}$ uptake and MIB-1 monoclonal antibody cytochemical staining of nuclei (4). In another study, Buck et al related the increased ${ }^{18} \mathrm{~F}-\mathrm{FLT}$ uptake exclusively to malignant tumors (5). However, to the best of our knowledge, ${ }^{18}$ F-FLT has not been investigated comprehensively in clinical settings. We recently conducted a randomized, blinded, prospective multicenter clinical trial (MCCT) on PET/CT of pulmonary nodules using both ${ }^{18} \mathrm{~F}-\mathrm{FDG}$ and ${ }^{18} \mathrm{~F}$-FLT. The aims of this trial were (a) to verify the diagnostic performance of ${ }^{18} \mathrm{~F}$ FDG, ${ }^{18} \mathrm{~F}-\mathrm{FLT}$, and dual-tracer imaging, (b) to determine the possible clinical advantage of dual-tracer PET/CT, and (c) to test the reliability and objectivity of the diagnostic criteria established in the trial.

\section{MATERIALS AND METHODS}

\section{General Description of Study}

This 2-y multicenter clinical trial was designed by a special task group consisting of nuclear medicine physicians, statisticians, Good Clinical Practice (GCP) staff, and law workers. The first half-year was dedicated to the staff training, preparation and printing of data sheets, case reporting forms, standard of operational 
protocol (SOP), and other documents. The clinical imaging and data analysis took place in the next $1.5 \mathrm{y}$. Seven medical centers participated in the trial. One center acted as the organizer, taking the responsibility for collecting, verifying, and centrally processing data. The other 6 centers imaged the patients. To ensure the integrity of the data and the objectivity of the evaluation, the images acquired and processed using the standardized protocols and quality control-as well as the data recorded in the normalized forms-were collected by the organizing center, where a workstation had been installed for centralized processing. The images were interpreted in a randomized, blinded, collective reading, and the consensus diagnosis was compared with the standard of truth. The study protocol had been approved by the regional or hospital medical ethic committees.

\section{Patient Enrollment}

Patients entered the study in a sequential order. The inclusion criteria included (a) radiologic evidence of a pulmonary nodule or nodules $(\leq 3)$, (b) no definite diagnosis, (c) no specific treatment before the study, (d) no significant dysfunction or disorder of major organs as suggested by laboratory or clinical signs (e.g., blood glucose $<6.16 \mathrm{mmol} / \mathrm{L}$, alanine aminotransferase $<40 \mathrm{U} / \mathrm{L}$, and urea $<7.50 \mathrm{mmol} / \mathrm{L}$ ), (e) willingness to follow the study protocols and to give written consent for participation in the trial, and (f) possible clinical outcome expected within the foreseeable duration of follow-up. The age and sex of the patients were of no concern in this trial.

The exclusion criteria included (a) diagnosis already defined, (b) severely ill or with metabolic abnormalities such as hyperglycemia, (c) unlikely to comply with the study protocols, and (d) unable to provide necessary clinical data. A patient's data would be excluded from the final analysis if a question existed on the quality of either radiotracer or PET/CT scan or the diagnosis was still in doubt at the time of final collective image reading.

\section{Equipments and Acquisition Parameters}

A similar model of PET/CT scanner (Discovery ST; GE Healthcare) and the same kinds of cyclotron (MiniTrace; GE Healthcare) and synthesizer (TracerLab $\mathrm{Fx}_{\mathrm{FN}}$; GE Healthcare) were used for $\mathrm{PET} / \mathrm{CT}$ and radiotracer production in this trial. An obligatory standardized quality-control program was followed by all imaging centers and was subject to the organizer's inspections. To protect the patients from undue radiation dosage, a low-dose CT scan was acquired with the following settings: $120 \mathrm{kV}, 100 \sim 250 \mathrm{mAs}$ with automatic adjustment, $0.8-\mathrm{s}$ rotation, $1.25-\mathrm{mm}$ collimation, and a pitch varied according to the geometry of the CT detector $(4,8$, or 16 slices). The PET scanner has a $15.7-\mathrm{cm}$ axial field width and a spatial resolution of 4-mm full width at half maximum at $1 \mathrm{~cm}$ from the center. PET images were acquired in 3-dimensional mode, with 2.5 min per bed and 3 or 4 bed positions covering the entire chest. In some cases, whole-body ${ }^{18}$ F-FDG imaging was performed from the bottom of the pelvis to the chin. The images were reconstructed in a Fourier rebinning iterative algorithm. Delayed imaging with similar acquisition parameters was recommended whenever possible.

\section{Radiopharmaceuticals}

Both ${ }^{18}$ F-FDG and ${ }^{18}$ F-FLT were automatically synthesized in each imaging center. The raw materials and agents for the synthesis were purchased by the organizer from the same supplier and delivered to each center. Both syntheses and quality control for every preparation of the pharmaceuticals strictly followed the
SOPs and were subject to inspection. The labeling yield, radiochemical purity, and specific radioactivity of the product were checked and recorded after each production. The products had to meet certain criteria-for example, the radiochemical yield must be $>10 \%$ and the radiochemical purity must be $>95 \%$, to be used for imaging.

\section{Imaging Protocols}

Each patient was imaged twice using ${ }^{18} \mathrm{~F}-\mathrm{FDG}$ and ${ }^{18} \mathrm{~F}-\mathrm{FLT}$ within $7 \mathrm{~d}$. The order of ${ }^{18} \mathrm{~F}-\mathrm{FDG}$ or ${ }^{18} \mathrm{~F}$-FLT scanning of each patient was determined randomly by a binary code produced by a computer. The patient was asked to fast over $4 \mathrm{~h}$ and to rest for 15 min before administration of $300 \sim 400 \mathrm{MBq}$ radioactive tracers. The images were acquired at $60 \mathrm{~min}$ after injection. On the basis of the clinical situation and the patient's agreement, some patients had a delayed scan at $120 \mathrm{~min}$ after injection. Within $7 \mathrm{~d}$, the whole procedure was repeated using the alternative radiopharmaceutical.

\section{Image Interpretation}

The CT images were displayed as 5-mm cross-axial slices. The morphologic features of the nodule(s) were checked-such as the size, density, cavity, calcification, notch on margin, spiculated margin or plural contraction, and so forth-and the CT value was assessed. The PET images were visually inspected with the maximum standardized uptake value $\left(\mathrm{SUV}_{\mathrm{MAX}}\right)$ determined from a circular region of interest (ROI) over the entire lesion. The uptake of a lesion was also scored in the following manner. In the case of ${ }^{18} \mathrm{~F}-\mathrm{FDG}, 0=$ no uptake; $1=$ uptake lower than that of the mediastinum; 2 = uptake equal to or greater than that of the mediastinum but lower than that of the liver; 3 = obvious uptake higher than that of the liver; and $4=$ very strong uptake. For ${ }^{18} \mathrm{~F}-\mathrm{FLT}, 0=$ no uptake, $1=$ barely visible uptake, $2=$ uptake lower than half the value of the thoracic vertebrae, $3=$ obvious uptake similar to that of the vertebrae, and $4=$ very high uptake.

The differential threshold for malignancy was set as $\mathrm{SUV}_{\mathrm{FDG}} \geq$ 2.5 , score $_{\mathrm{FDG}} \geq 2$ and $\mathrm{SUV}_{\mathrm{FLT}} \geq 1.4$, score $_{\mathrm{FLT}} \geq 1$. A lesion would be classified as malignant if uptake of both ${ }^{18} \mathrm{~F}-\mathrm{FDG}$ and ${ }^{18} \mathrm{~F}-\mathrm{FLT}$ or SUV and score were above the threshold, and the uptake of ${ }^{18} \mathrm{~F}$-FLT was lower than that of ${ }^{18} \mathrm{~F}$-FDG.

In cases of $>1$ lesion, the maximum scores and SUVs assessed among all lesions were chosen as the representative ones. The results in this trial were presented thereafter on a patient basis rather than on a lesion basis.

\section{Data Collection and Verification}

The original copies of working sheet and data record for each patient were sealed individually and, along with each patient's image data, sent to the organizing center over the Internet or by means of a CD-ROM. All sets of the serially numbered forms were required to be sent back to the organizing center whether they were or were not used. No correction or modification was allowed on the original records. After completion of the trial, the following datasets were collected from every center:

- Two original copies of PET/CT working sheet, one for each imaging-session.

- Two packages of raw image data from the dual-tracer PET/ CT imaging.

- The original follow-up records, with the date and type of surgical procedure and the pathologic diagnosis or the date and findings on follow-up. 
- The original copies of radiopharmaceutical production sheet, with the information on the production, quality control, and the raw material and agents used for each synthesis.

- The signed consent form from every subject.

- A summary of all cases, successful or failed, with the relevant information and explanations.

- A summary of the execution of the MCCT by each imaging center.

A group of physicians, physicists, radiochemists, administrators, and inspectors in the organizing center verified all data before further processing. Any noncompliance with the MCCT protocols resulted in exclusion of the patient's data. Forty patients were eventually excluded from the final analysis because of unsatisfactory image quality $(n=5)$, failure in ${ }^{18}$ F-FLT synthesis $(n=29)$, or incomplete follow-up data $(n=6)$. Only 55 patients passed the data verification in the final analysis.

\section{Collective Image Reading}

Two sessions of blinded, collective image reading were carried out in this trial. The first session was organized in the sixth month after the initiation of the trial with 3 independent readers who had a CT or PET professional background. The purpose of the first reading was to verify the interpretation criteria. The final reading session took place on completion of the trial with an expanded team of readers. Only the results of the final reading session were analyzed and reported in this article.

Nine readers took part in the final collective reading. Four had professional CT backgrounds and the other 5 had professional nuclear medicine backgrounds. All readers were responsible for the primary PET/CT image interpretation in their own imaging centers. They had 1 4 y working experience with PET/CT when the trial began.

In the collective reading, the images were reconstructed and assessed using the central workstation. Every patient's images were read 7 times using different strategies- ${ }^{18} \mathrm{~F}-\mathrm{FDG},{ }^{18} \mathrm{~F}-\mathrm{FLT}$, CT alone, ${ }^{18} \mathrm{~F}-\mathrm{FDG}+{ }^{18} \mathrm{~F}-\mathrm{FLT},{ }^{18} \mathrm{~F}-\mathrm{FDG}+\mathrm{CT},{ }^{18} \mathrm{~F}-\mathrm{FLT}+\mathrm{CT}$ in pairs, and, finally, the combination of ${ }^{18} \mathrm{~F}-\mathrm{FDG}+{ }^{18} \mathrm{~F}-\mathrm{FLT}+\mathrm{CT}$. In each round of reading, the present order of the patients' images was randomized and the heading of images was masked before viewing. The readers read all images unaware of any patient's information. The images were projected onto a screen. The display window and angles were adjusted as reader(s) requested assistance from an independent operator. No discussion was allowed among readers; each reader had to make his or her own judgment on each subject and score the images. The recording sheet of every reader was collected before the next round of reading. The imaging diagnosis was determined by a consensus reached by at least 5 of 9 readers, and the corresponding score was determined by averaging. A ratio of $\mathrm{SUV}_{\mathrm{FLT}} / \mathrm{SUV}_{\mathrm{FDG}}$ was also calculated in the collective reading. On completion of the final collective reading, the data were available to the readers, and the results were statistically analyzed in light of the standard of truth.

\section{Endpoint and Standard of Truth}

The endpoint of this trial was determined as either the pathologic evidence obtained from surgical processes or the clinical conclusion derived from the therapeutic response or from imaging or laboratory findings in follow-up over 1 y after imaging. Therefore, the standard of truth was the histologic diagnosis or the validated clinical evidence derived at the end of 12-mo follow-up period.

\section{Statistical Analysis}

Commercial (SPSS11.0) and dedicated (MINITABLE for 6 Sigma; GE Healthcare) software packages were used for the statistical analysis. The comparison on the diagnostic performances of image interpretation strategies and the correlation between SUVs and scores were analyzed. A statistician took an active part in the design, data check, and final analysis in this trial.

\section{RESULTS}

\section{Clinical Trial}

The first patient in this MCCT was imaged in January 2006, and the follow-up of the last patient was completed by June 2007 . Among 55 patients entering the final analysis (Table 1), 33 were males and 22 were females (age range, 17- to 82 -y-old). There were 28 patients with a solitary pulmonary nodule and 27 with 2 or 3 nodules. The size of the nodules ranged from 6 to $\sim 110 \mathrm{~mm}$, with the majority of lesions $<30 \mathrm{~mm}(n=35)$. Because the 6 imaging centers covered wide geographic areas in China, with populations of different living habits and environment, the underlining diseases of our subjects were quite heterogeneous. The final diagnosis included 16 lung cancers, 16 tuberculoses, and 23 other benign lesions (inflammation, pseudotumor, granuloma, and other benign conditions). The diagnosis was confirmed via surgical processes (operation or biopsy) in 27 patients or via other clinical processes in 28 patients. No side effects were reported with either radiopharmaceutical or in PET/CT scanning.

\section{${ }^{18}$ F-FDG Images}

A positive uptake of ${ }^{18} \mathrm{~F}-\mathrm{FDG}$ was noted in nearly all lesions (Fig 1A). The uptake varied in intensity, with a $\mathrm{SUV}_{\text {MAX }}$ of $0.75 \sim 16.0$ and corresponding scores of $0 \sim 4$. The mean SUV $\pm \mathrm{SD}$ in patients with a malignancy $(n=$ 16) was $8.13 \pm 3.69$ (range, $2.0 \sim 16.0$ ), and the score was $3.12 \pm 0.86$ (range, $1.22 \sim 4.0$ ), higher than that of tuberculoses (TB) $(n=16)$ and other benign diseases $(n=23)$. The corresponding values in the latter 2 groups were $5.71 \pm$ 2.90 (range, $1.24 \sim 12.79$ ), $2.73 \pm 0.70$ (range, 0.89 3.89), and $4.71 \pm 0.74$ (range, $0.75 \sim 16.0$ ), $1.96 \pm 1.33$ (range, $0.61 \sim 4.0)$, respectively. ANOVA revealed a significant difference between groups in both $\operatorname{SUV}\left(\mathrm{F}_{2,52}=4.583\right.$, $P=0.015)$ and score $\left(\mathrm{F}_{2,52}=6.338, P=0.006\right)$. The Games-Howell test suggested that significant difference existed only between tumor and other benign lesions in both SUV $(P=0.021)$ and score $(P=0.005)$. There was a nonlinear correlation between the ${ }^{18} \mathrm{~F}-\mathrm{FDG} \mathrm{SUV}_{\mathrm{MAX}}$ and the ${ }^{18} \mathrm{~F}$-FDG scores $\left(r^{2}=0.51, P=0.000\right.$; Fig. 2$)$.

\section{${ }^{18}$ F-FLT Images}

In general, the uptake of ${ }^{18} \mathrm{~F}$-FLT by a pulmonary lesion was lower than that of ${ }^{18} \mathrm{~F}-\mathrm{FDG}$ (Fig. 1B). The higher uptake by liver and bone marrow of vertebrae and ribs made the detection of small lesion(s) by ${ }^{18} \mathrm{~F}$-FLT more difficult. The SUV $\mathrm{MAX}$ and scores in malignancies were $3.54 \pm 1.98$ (range, 0.9 9.40) and $1.64 \pm 1.14$ (range, $0 \sim 3.81$ ). The values in TB were $1.65 \pm 0.99$ (range, $0 \sim 3.3$ ) 
TABLE 1

Summary of Subjects

\begin{tabular}{|c|c|c|c|c|c|c|c|c|}
\hline Patient & Sex & Age (y) & No. of nodules & Size (mm) & Diagnosis & SUV $_{\mathrm{FDG}}$ & SUV $_{\mathrm{FLT}}$ & FLT/FDG \\
\hline 1 & $\mathrm{~F}$ & 42 & 1 & $20 \times 21$ & Ad & 9.7 & 4.13 & 0.43 \\
\hline 2 & $\mathrm{~F}$ & 53 & 2 & $20 \times 20$ & TB & 4.45 & 2.23 & 0.50 \\
\hline 3 & $M$ & 29 & 3 & $22 \times 23$ & TB & 5.8 & 2.3 & 0.40 \\
\hline 4 & $\mathrm{~F}$ & 17 & 3 & $37 \times 49$ & TB & 7 & 2.68 & 0.38 \\
\hline 5 & $\mathrm{~F}$ & 44 & 2 & $10 \times 10$ & $\mathrm{SN}$ & 3 & 4.2 & 1.40 \\
\hline 6 & M & 38 & 3 & $30 \times 50$ & TB & 4.8 & 1.6 & 0.33 \\
\hline 7 & M & 81 & 2 & $15 \times 32$ & Ad & 10.9 & 9.4 & 0.86 \\
\hline 8 & M & 45 & 1 & $15 \times 10$ & SN & 6.56 & 2.04 & 0.31 \\
\hline 9 & $M$ & 76 & 1 & $25 \times 15$ & $\mathrm{Sq}$ & 8 & 3.8 & 0.48 \\
\hline 10 & $\mathrm{~F}$ & 48 & 2 & $29 \times 41$ & IN & 2.2 & 0.8 & 0.36 \\
\hline 11 & $\mathrm{~F}$ & 46 & 3 & $19 \times 33$ & GN & 3.6 & 1.32 & 0.37 \\
\hline 12 & $\mathrm{~F}$ & 65 & 1 & $15 \times 14$ & TB & 5 & 1.3 & 0.26 \\
\hline 13 & M & 32 & 2 & NA & TB & 4 & 1.4 & 0.35 \\
\hline 14 & $\mathrm{M}$ & 58 & 2 & $8 \times 10$ & Ad & 2 & 0.9 & 0.45 \\
\hline 15 & $M$ & 69 & 3 & $28 \times 26$ & Ad & 10.8 & 4.6 & 0.43 \\
\hline 16 & $\mathrm{~F}$ & 73 & 1 & $17 \times 12$ & $\mathrm{SN}$ & 2.2 & 2.2 & 1.00 \\
\hline 17 & $M$ & 35 & 3 & $26 \times 23$ & TB & 7.1 & 1.33 & 0.19 \\
\hline 18 & $M$ & 40 & 3 & $35 \times 33$ & WG & 16 & 4.73 & 0.30 \\
\hline 19 & $M$ & 46 & 1 & $15 \times 26$ & TB & 1.24 & 0 & 0 \\
\hline 20 & M & 58 & 1 & $19 \times 18$ & Ad & 5 & 2.5 & 0.50 \\
\hline 21 & $M$ & 68 & 1 & $15 \times 20$ & SN & 1.9 & 0.5 & 0.26 \\
\hline 22 & $M$ & 43 & 3 & $25 \times 31$ & TB & 12.79 & 3.3 & 0.26 \\
\hline 23 & $M$ & 68 & 1 & $20 \times 30$ & SN & 11.06 & 5.1 & 0.46 \\
\hline 24 & $M$ & 63 & 2 & $70 \times 40$ & IN & 3.9 & 4.4 & 1.13 \\
\hline 25 & $\mathrm{~F}$ & 60 & 1 & $55 \times 40$ & TB & 3.02 & 1.11 & 0.37 \\
\hline 26 & $\mathrm{~F}$ & 37 & 2 & $25 \times 30$ & MT & 6 & 2.7 & 0.45 \\
\hline 27 & $M$ & 31 & 1 & $15 \times 20$ & SN & 3.92 & 1.42 & 0.36 \\
\hline 28 & $\mathrm{~F}$ & 35 & 1 & NA & TB & 3.8 & 1.47 & 0.39 \\
\hline 29 & $F$ & 43 & 1 & $10 \times 10$ & $\mathrm{SN}$ & 0.75 & 0.87 & 1.10 \\
\hline 30 & $\mathrm{~F}$ & 71 & 3 & $35 \times 40$ & IN & 8 & 3.1 & 0.39 \\
\hline 31 & $M$ & 50 & 1 & $75 \times 110$ & Ad & 16 & 4.73 & 0.30 \\
\hline 32 & M & 64 & 1 & $12 \times 13$ & Ad & 3.7 & 1.7 & 0.46 \\
\hline 33 & M & 46 & 3 & $55 \times 40$ & $\mathrm{BA}$ & 10 & 3.1 & 0.31 \\
\hline 34 & $\mathrm{~F}$ & 59 & 1 & $15 \times 20$ & $\mathrm{SN}$ & 7 & 0 & 0 \\
\hline 35 & $\mathrm{~F}$ & 67 & 1 & $15 \times 10$ & TB & 3.9 & 0 & 0 \\
\hline 36 & $\mathrm{~F}$ & 53 & 2 & $22 \times 30$ & BA & 7.3 & 2.9 & 0.40 \\
\hline 37 & $M$ & 66 & 2 & $11 \times 13$ & IN & 1.6 & 2.1 & 1.31 \\
\hline 38 & M & 70 & 2 & $25 \times 30$ & Ad & 6 & 3.2 & 0.53 \\
\hline 39 & $M$ & 62 & 1 & $15 \times 10$ & TB & 4.1 & 1.4 & 0.34 \\
\hline 40 & $M$ & 71 & 2 & $45 \times 38$ & Ad & 10.5 & 4.3 & 0.41 \\
\hline 41 & $M$ & 82 & 1 & NA & TB & 10.2 & 3.2 & 0.31 \\
\hline 42 & $\mathrm{~F}$ & 58 & 1 & $23 \times 23$ & SN & 1.83 & 2.1 & 1.15 \\
\hline 43 & $\mathrm{~F}$ & 53 & 3 & $21 \times 19$ & SN & 1.9 & 1.8 & 0.95 \\
\hline 44 & $M$ & 36 & 2 & $15 \times 20$ & IN & 2 & 2.1 & 1.05 \\
\hline 45 & $\mathrm{~F}$ & 54 & 1 & $10 \times 13$ & TB & 8.78 & 2.4 & 0.27 \\
\hline 46 & $\mathrm{~F}$ & 49 & 1 & $20 \times 10$ & GN & 3.25 & 1 & 0.31 \\
\hline 47 & $M$ & 53 & 1 & $6 \times 5$ & Ad & 3 & 1.7 & 0.57 \\
\hline 48 & M & 80 & 2 & $23 \times 30$ & $\mathrm{Sq}$ & 10 & 5 & 0.50 \\
\hline 49 & $M$ & 56 & 1 & $17 \times 12$ & IN & 7.7 & 0.32 & 0.04 \\
\hline 50 & $M$ & 76 & 2 & $28 \times 20$ & SN & 9.08 & 0.76 & 0.08 \\
\hline 51 & $M$ & 70 & 1 & $29 \times 33$ & BA & 11.24 & 2 & 0.18 \\
\hline 52 & $M$ & 77 & 1 & $61 \times 28$ & TB & 5.36 & 0.68 & 0.13 \\
\hline 53 & M & 64 & 1 & $31 \times 26$ & IN & 5.78 & 3.4 & 0.59 \\
\hline 54 & $\mathrm{~F}$ & 35 & 3 & $18 \times 13$ & SN & 0.75 & 0.8 & 1.07 \\
\hline 55 & $\mathrm{~F}$ & 55 & 1 & $25 \times 26$ & PT & 4.2 & 0.42 & 0.10 \\
\hline
\end{tabular}

$\mathrm{Ad}=$ adenocarcinoma; $\mathrm{TB}=$ tuberculosis; $\mathrm{SN}=$ stable nodule (no change after $1 \mathrm{y}$ ); $\mathrm{Sq}=$ squamous cancer; IN = inflammatory nodule (response to antibiotics); GN = granulomatous nodule; NA = not assessable; WG = Wegener's granuloma; MT = metastatic tumor; $\mathrm{BA}=$ bronchioalveolar carcinoma; $\mathrm{PT}=$ pseudotumor. 


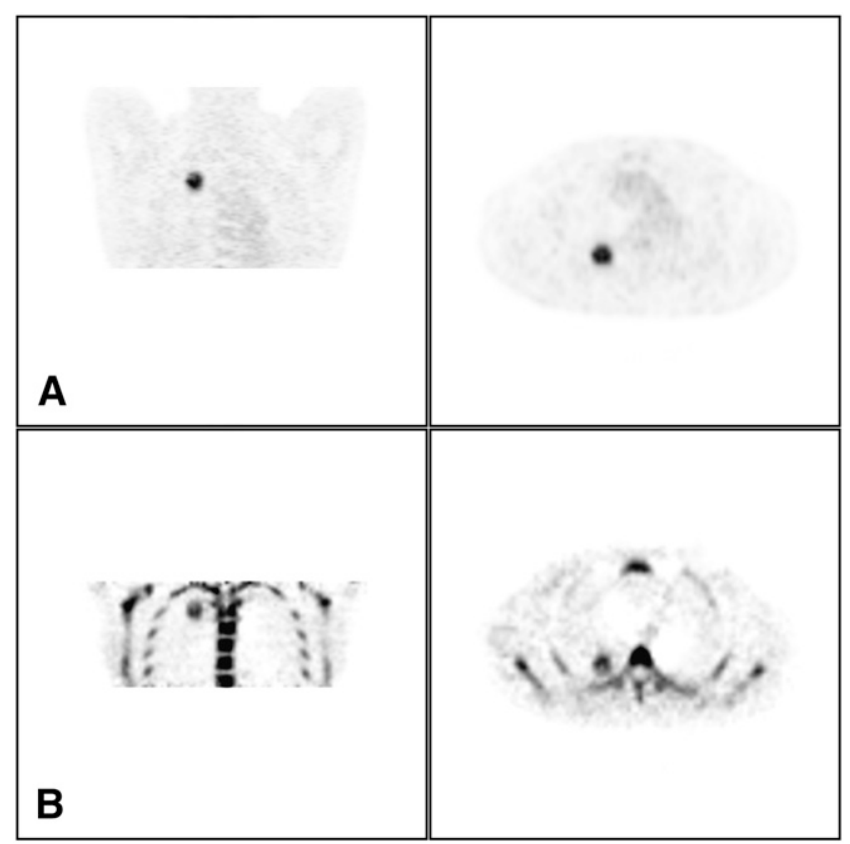

FIGURE 1. Patient 15 (male, 69-y-old) with adenocarcinoma of right lung $\left({ }^{18} \mathrm{~F}-\mathrm{FDG} /{ }^{18} \mathrm{~F}-\mathrm{FLT}=0.43\right)$. (A) ${ }^{18} \mathrm{~F}-\mathrm{FDG}$ PET images are displayed in coronal and transaxial slices $\left(\mathrm{SUV}_{\mathrm{MAX}}=10.8\right)$. (B) ${ }^{18} \mathrm{~F}-\mathrm{FLT}$ PET in the same patient $\left(\mathrm{SUV}_{\mathrm{MAX}}=4.6\right)$. Difference on image quality was noticeable.

and $1.21 \pm 0.66$ (range, $0 \sim 1.61$ ), and in other benign lesions, the values were $1.56 \pm 1.60$ (range, $0-5.10$ ) and $0.80 \pm 0.81$ (range, $0.44 \sim 2.17$ ), respectively. ANOVA also indicated a significant difference among groups in SUV $\left(\mathrm{F}_{2,52}=7.119, P=0.002\right)$ and score $\left(\mathrm{F}_{2,52}=6.786, P=\right.$ 0.005). The Games-Howell test confirmed the significance of SUV difference between tumor, TB $(P=0.007)$, and other benign lesions $(P=0.033)$ but not between the 2 benign groups, whereas the score differed only between tumor and other benign diseases $(P=0.041)$. A linear correlation was noted between the ${ }^{18} \mathrm{~F}-\mathrm{FLT}$ SUV $\mathrm{MAX}$ and ${ }^{18}$ F-FLT scores $\left(r^{2}=0.59, P=0.000\right.$; Fig. 3$)$.

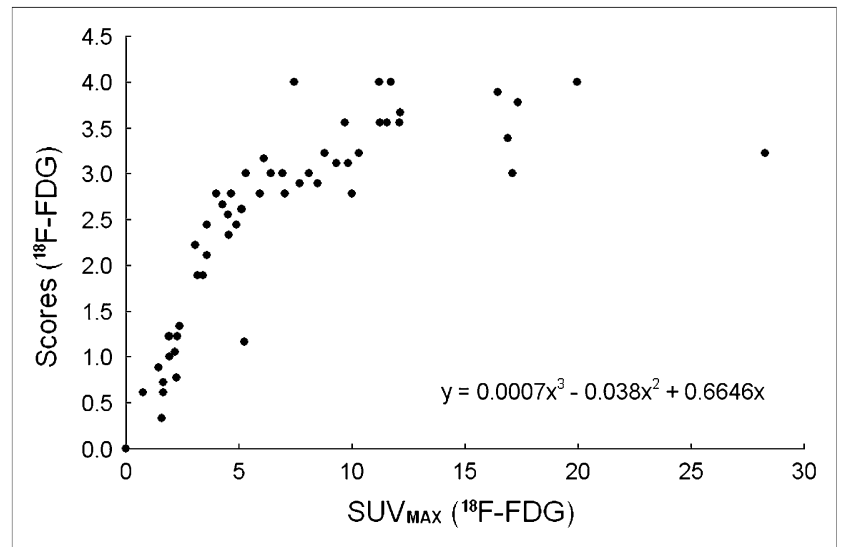

FIGURE 2. Nonlinear correlation existed between SUV and scores of ${ }^{18} \mathrm{~F}-\mathrm{FDG}$.

\section{${ }^{18} \mathrm{~F}-\mathrm{FLT}$ and ${ }^{18} \mathrm{~F}-\mathrm{FDG}$ Ratios}

Interestingly, the ratio between ${ }^{18} \mathrm{~F}$-FLT $\mathrm{SUV}_{\mathrm{MAX}}$ and ${ }^{18} \mathrm{~F}-\mathrm{FDG} \mathrm{SUV}_{\mathrm{MAX}}\left({ }^{18} \mathrm{~F}-\mathrm{FLT} /{ }^{18} \mathrm{~F}-\mathrm{FDG}\right)$ was found to be more accurate than any other criteria in the separation of different subgroups of patients (Fig. 4). Among 29 lesions with an ${ }^{18} \mathrm{~F}-\mathrm{FLT} /{ }^{18} \mathrm{~F}-\mathrm{FDG}$ ratio $<0.40,14$ were TB (Fig. 5), 12 were inflammation, and only 3 were malignant. All lesions with an ${ }^{18} \mathrm{~F}-\mathrm{FLT} /{ }^{18} \mathrm{~F}-\mathrm{FDG}$ ratio $>0.90$ were inflammation (Fig. 6), whereas in 17 lesions with an ${ }^{18} \mathrm{~F}-\mathrm{FLT} /{ }^{18} \mathrm{~F}-\mathrm{FDG}$ ratio between 0.40 and $0.90,13$ were malignant. The difference in the ${ }^{18} \mathrm{~F}-\mathrm{FLT} /{ }^{18} \mathrm{~F}$-FDG ratio between the 3 subgroups was statistically significant $\left(\mathrm{F}_{2,49}=5.361, P=\right.$ 0.008). The Games-Howell test indicated a significant difference between tumor and TB $(0.45 \pm 0.146$ vs. $0.27 \pm$ $0.140, P=0.005)$ and between TB and other benign disease $(0.61 \pm 0.450, P=0.007)$ but not between tumors and inflammation $(P=0.269)$, probably due to a rather large SD in the latter subgroup of patients.

\section{Dual-Phase ${ }^{18}$ F-FDG and ${ }^{18}$ F-FLT Scans}

Only 34 patients had delayed ${ }^{18} \mathrm{~F}-\mathrm{FDG}$ and 25 patients had delayed ${ }^{18}$ F-FLT $(n=25)$ imaging. The change in the radiotracer uptake between early and delayed imaging varied unpredictably. In delayed ${ }^{18}$ F-FDG imaging, 5 of 6 tumors, 9 of $12 \mathrm{~TB}$, and 9 of 16 inflammations had their SUVs increased in delayed imaging, whereas decreased SUVs were noted in 1 of 6 tumors, 3 of 12 TB, and 4 of 16 inflammations. The mean $\pm \mathrm{SD}$ of delayed $\mathrm{SUV}_{\mathrm{FDG}}$ was $8.97 \pm 4.26$ in tumor, $6.69 \pm 3.04$ in $\mathrm{TB}$, and $4.76 \pm$ 3.41 in inflammation. The $\Delta$ SUV was $24 \% \pm 31 \%, 20.9 \% \pm$ $35.3 \%$, and $4.52 \% \pm 26.95 \%$, respectively. Increased ${ }^{18} \mathrm{~F}_{-\mathrm{FLT}_{\text {SUV }}}$ was noted in 3 of 7 tumors, 3 of $8 \mathrm{~TB}$, and 2 of 10 inflammations. Four tumors, 5 TB, and 6 inflammations had decreased $\mathrm{SUV}_{\mathrm{FLT}}$ in delayed scanning. The delayed ${ }^{18} \mathrm{~F}_{-F} \mathrm{FLT}_{\text {SUV }}$ was $4.18 \pm 2.71,1.59 \pm 1.10$, and $1.34 \pm 0.91$, and the $\Delta$ SUV was $-0.09 \% \pm 13.31 \%$, $-12.3 \% \pm 20.48 \%$, and $10.6 \% \pm 41.35 \%$ in the 3 subgroups of patients, respectively. Statistically, no difference

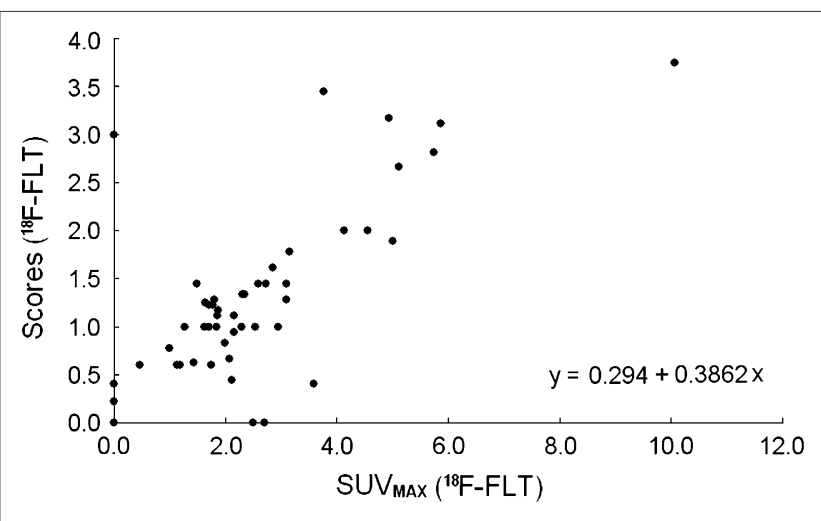

FIGURE 3. Linear correlation existed between SUV MAX and scores of ${ }^{18} \mathrm{~F}-\mathrm{FLT}$. 


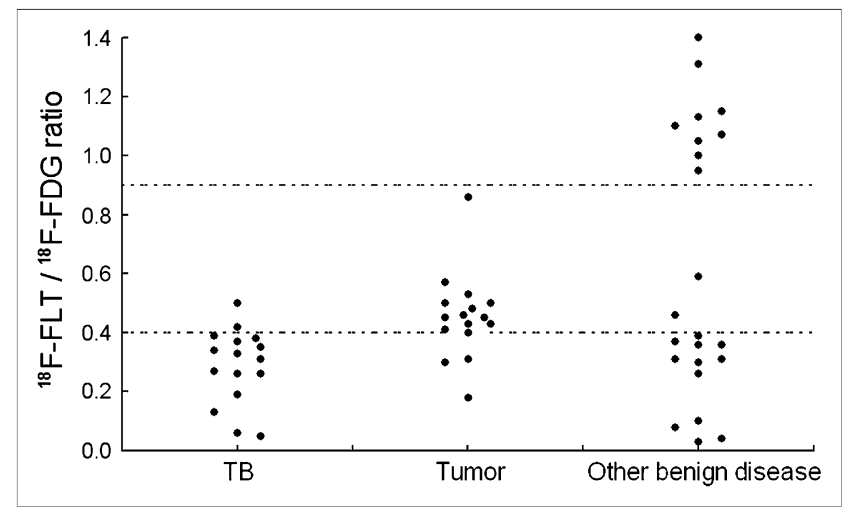

FIGURE 4. Subgroups of patients with TB, tumor, and other benign diseases could be separated using an ${ }^{18} \mathrm{~F}-\mathrm{FLT} /{ }^{18} \mathrm{~F}-\mathrm{FDG}$ SUV ratio of $0.40 \sim 0.90$. Thirteen of 16 tumors were in this range; only 2 TB and 2 inflammations were false-positive. Fourteen TB and 12 inflammations had ${ }^{18} \mathrm{~F}-\mathrm{FLT} /{ }^{18} \mathrm{~F}-\mathrm{FDG}$ ratios lower than 0.39 . Nine patients had inflammatory lesions with ${ }^{18} \mathrm{~F}-\mathrm{FLT} /{ }^{18} \mathrm{~F}-\mathrm{FDG}$ ratios higher than 0.95 .

in $\triangle$ SUV could be demonstrated between groups using either radiotracer.

\section{Diagnostic Performance of Different Imaging Strategies}

In the final collective image reading, ${ }^{18} \mathrm{~F}-\mathrm{FDG}$ PET correctly detected 14 of 16 malignant lesions. However, falsepositive scans were noted in 16 of 39 benign cases. The sensitivity of ${ }^{18}$ F-FLT PET was lower (11/16) and so was its false-positive rate (9/39). The low-dose CT alone had a diagnostic efficiency similar to that of ${ }^{18} \mathrm{~F}-\mathrm{FLT}$ (sensitivity, 11/16; specificity, 29/39). When the images were read in

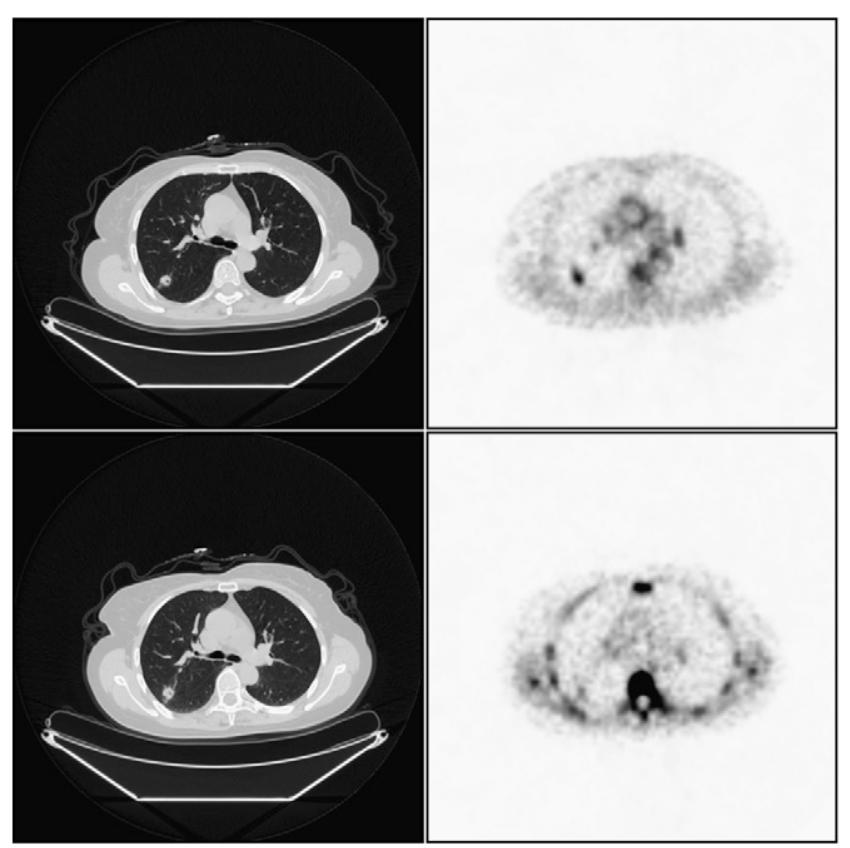

FIGURE 5. Patient 12 (female, 65-y-old) with $1.5 \times 1.4 \mathrm{~cm}$ lesion at right upper lobe. Confirmed diagnosis was tuberculoses. SUV $\mathrm{FDG}=5.0, \mathrm{SUV}_{\mathrm{FLT}}=1.3$, and ${ }^{18} \mathrm{~F}-\mathrm{FLT} /{ }^{18} \mathrm{~F}-\mathrm{FDG}=$ $26 \%$.

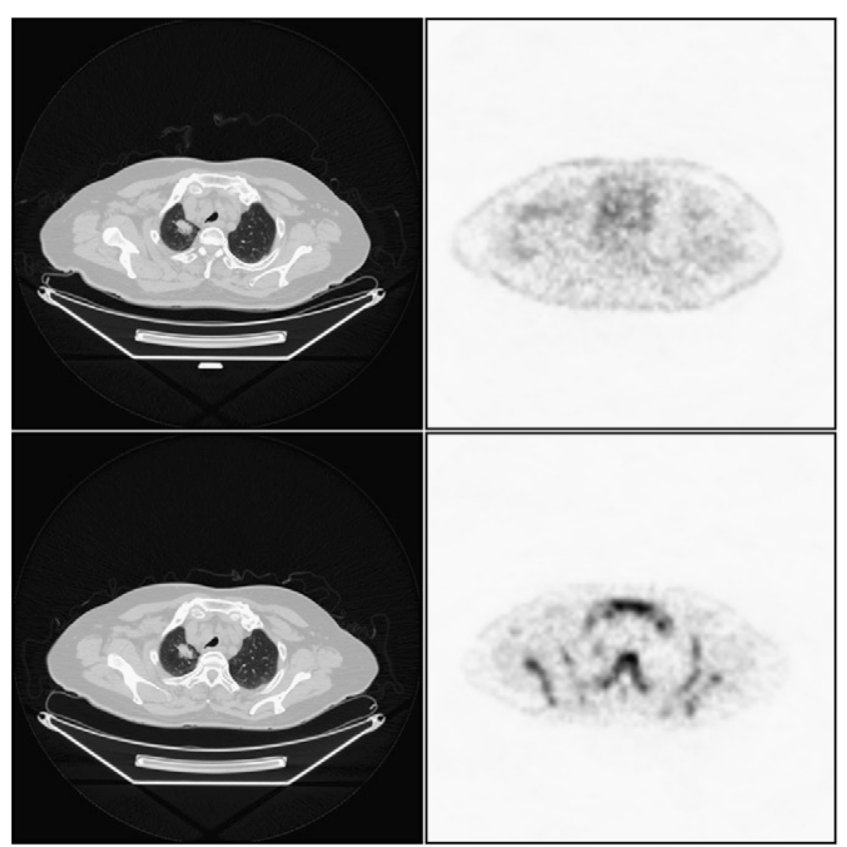

FIGURE 6. Patient 16 (female, 73 -y-old) with $1.7 \times 1.2 \mathrm{~cm}$ confirmed inflammatory nodule in right upper lung. SUV $\mathrm{FDG}_{\mathrm{F}}=$ 2.2, $\mathrm{SUV}_{\mathrm{FLT}}=2.2$, and ${ }^{18} \mathrm{~F}-\mathrm{FLT} /{ }^{18} \mathrm{~F}-\mathrm{FDG}=100 \%$.

pairs-such as ${ }^{18} \mathrm{~F}$-FDG $+\mathrm{CT},{ }^{18} \mathrm{~F}-\mathrm{FLT}+\mathrm{CT}$, or ${ }^{18} \mathrm{~F}$-FDG + ${ }^{18} \mathrm{~F}-\mathrm{FLT}$ - the specificity improved, especially when ${ }^{18} \mathrm{~F}-$ FLT was read with ${ }^{18} \mathrm{~F}-\mathrm{FDG}$. CT showed its value in cases of solitary pulmonary nodule (SPN) $<10 \mathrm{~mm}$ or with very low uptake when reading became more difficult. The diagnostic performance was obviously improved when the 3 sets of images were read together, resulting in the highest sensitivity, specificity, and accuracy (Table 2). The $\chi^{2}$ test based on expected correct-wrong reading suggested that at least 1 strategy had an important influence on the overall diagnostic performance $\left(\chi^{2}=18.225, P=0.006\right)$ and that the paired ${ }^{18} \mathrm{~F}$-FDG $+{ }^{18} \mathrm{~F}$-FLT had the highest correct reading over other strategies; therefore, this strategy probably had the highest contribution to the interpretation accuracy (Table 3).

\section{Cases with False Result}

The data of 3 patients with lung malignancy were misinterpreted as benign in the collective reading. All were males (age range, 46- to 70-y-old). Bronchioalveolar cell carcinoma was pathologically proven in 2 patients. Careful reevaluation of the other case found an out-of-record "antitumor" therapy of unknown nature before the patient entered the study; therefore, the therapeutic effect on his lesions at the time of imaging could not be excluded.

False-positive results were encountered in 2 inflammatory nodules and 2 tuberculoses. One patient underwent surgery on the basis of the ${ }^{18} \mathrm{~F}-\mathrm{FLT} /{ }^{18} \mathrm{~F}-\mathrm{FDG}$ PET result, to confirm a lesion of TB. The other patient with TB responded to anti-TB treatment. The lesions of other 2 patients revealed no change in follow-up without antitumor treatment. 
TABLE 2

Diagnostic Performance of Different Reading Strategies in 55 Patients

\begin{tabular}{llllcccc}
\hline Parameter (\%) & FDG & FLT & LDCT & FDG + CT & FLT + CT & FDG + FLT & FLT + FDG + CT \\
\hline Sensitivity & 87.5 & 68.75 & 68.75 & 87.50 & 75.0 & 81.25 & 100 \\
Specificity & 58.97 & 76.92 & 74.36 & 69.23 & 87.18 & 87.18 & 89.74 \\
Accuracy & 67.27 & 74.45 & 72.73 & 74.55 & 83.64 & 85.45 & 92.73 \\
PPV & 46.67 & 55.0 & 52.38 & 53.85 & 70.59 & 72.22 & 80.0 \\
NPV & 92.0 & 85.71 & 85.29 & 93.1 & 89.47 & 91.89 & 100
\end{tabular}

LDCT $=$ low-dose CT; PPV = positive predictive value; NPV = negative predictive value.

\section{DISCUSSION}

\section{Design of Trial}

The limitation of ${ }^{18} \mathrm{~F}-\mathrm{FDG}$ in the evaluation of lung tumors has been well documented. The reported positive predictive value of ${ }^{18} \mathrm{~F}$-FDG PET or PET/CT in pulmonary lesions was as low as $44.6 \%$ (6). ${ }^{18} \mathrm{~F}$-FLT was developed to reflect the proliferation rate of the lesions and to characterize malignant tumor growth $(7,8)$. The current dual-tracer study was designed to prove the following assumptions: (a) ${ }^{18} \mathrm{~F}$-FDG and ${ }^{18} \mathrm{~F}$-FLT provide information relating to different aspects of tumor biology; (b) dual-tracer imaging, with ${ }^{18} \mathrm{~F}$-FDG and ${ }^{18} \mathrm{~F}$-FLT complementary to each other, adds diagnostic confidence; and (c) the criteria in dual-tracer PET/CT image interpretation are objective, accurate, and easy to use. Enormous efforts were taken in this MCCT to avoid known bias that would affect the results.

\section{Comparison of ${ }^{18} \mathrm{~F}-\mathrm{FLT}$ and ${ }^{18} \mathrm{~F}-\mathrm{FDG}$}

In most of the cases, ${ }^{18} \mathrm{~F}$-FDG PET detected more lesions than ${ }^{18}$ F-FLT. The image quality of ${ }^{18}$ F-FDG was also superior with higher SUVs. The better image quality was believed to be due primarily to its comparatively lower background and more uniform tissue distribution. However, as well described by many authors, nonspecific uptake was noted in a number of benign lesions, especially in tuberculoses. Using $\mathrm{SUV}_{\mathrm{MAX}}=2.5$ as the differential threshold, ${ }^{18}$ F-FDG PET in our study had a fairly low specificity of $58.97 \%$ and an accuracy of $67.27 \%$. These figures were lower than those reported earlier (6). The major reason for the lower accuracy was believed to be the relatively higher proportion of $\mathrm{TB}$ among the studied subjects, resulting from a higher prevalence of TB in the districts covered by our imaging centers. Another factor to be considered was that most of our patients had multiple pulmonary nodules. It was recognized earlier that the false-positive rate of ${ }^{18} \mathrm{~F}$ FDG PET was higher in cases of multiple lung lesions (6).

In comparison with ${ }^{18} \mathrm{~F}$-FDG, ${ }^{18} \mathrm{~F}$-FLT images was more "noisy." The positive bone marrow of the thoracic cage interfered with the evaluation of intrathoracic lesions. In most cases, the uptake of ${ }^{18}$ F-FLT was lower than that of ${ }^{18} \mathrm{~F}$-FDG, which was in accordance with other reports. For example, Buck et al reported that ${ }^{18}$ F-FLT uptake was only $50 \%$ of ${ }^{18} \mathrm{~F}-\mathrm{FDG}$ uptake in positive nodal metastases of non-small cell lung cancer. ${ }^{18} \mathrm{~F}$-FLT had better specificity (76.92\% vs. $58.97 \%$ ) in our study, but the increased ${ }^{18} \mathrm{~F}$ FLT uptake was not "related exclusively to malignant tumors" as Buck et al. described (5). The uptake of ${ }^{18} \mathrm{~F}$ FLT was present to various degrees in many TB lesions and other benign lesions. This was not entirely unexpected because false-positive ${ }^{18} \mathrm{~F}$-FLT $\mathrm{PET}$ had been reported $(3,9)$. The mechanism of false-positive ${ }^{18} \mathrm{~F}$-FLT uptake is poorly understood. We noted that in our patients, the extent of ${ }^{18} \mathrm{~F}$-FLT accumulation in positive benign lesions did not correlate with that of the granulomatous tissue. In our previous in vitro study (Y Tan and J Tian, unpublished data, March 2006), the uptake of ${ }^{18}$ F-FLT by Escherichia coli in the growth phase was 6-fold higher than that of 3 tumor cell lines cultured simultaneously. Yap et al reported a false-positive ${ }^{18}$ F-FLT PET scan in a case of interstitial pneumonia with a Ki67 of $15 \%$ (9). Yamamoto et al. also reported a slightly increased $\mathrm{Ki67}(2.6 \% \pm 2.2 \%)$ in inflammatory cells (3). Thus, one may postulate that the ${ }^{18}$ F-FLT uptake could increase in response to active DNA synthesis in any tissue, including growing microbes. The effect of ${ }^{18}$ F-FLT transportation across cell membranes or a phosphorylation pathway other than thymidine kinase

TABLE 3

$\chi^{2}$ of Expected Correct and Wrong Reading Counts of Different Strategies

\begin{tabular}{|c|c|c|c|c|c|c|c|}
\hline Reading & FDG & FLT & LDCT & $\mathrm{FDG}+\mathrm{CT}$ & $\mathrm{FLT}+\mathrm{CT}$ & $F D G+F L T$ & FDG/FLT/CT \\
\hline Correct & 264 & 260 & 278 & 287 & 259 & 300 & 297 \\
\hline Wrong & 168 & 172 & 154 & 145 & 173 & 132 & 135 \\
\hline \multicolumn{8}{|c|}{$\begin{array}{l}\text { LDCT }=\text { low-dose CT. } \\
\chi^{2}=18.225, P=0.006\end{array}$} \\
\hline
\end{tabular}


1 should also be considered $(10,11)$. Because of its nontumor uptake, ${ }^{18} \mathrm{~F}$-FLT might not be suitable to work alone or to replace ${ }^{18} \mathrm{~F}-\mathrm{FDG}$ in the diagnosis or differentiation of pulmonary lesions.

One problem with ${ }^{18} \mathrm{~F}$-FLT was its fairly low production yield and success rate in synthesis $(7,12)$. With the commercially available automatic synthesizer used in our trial, the production succeeded only two thirds of the time, with an average yield of around $13 \%$. The unsatisfactory production efficacy negatively affected the clinical application of the tracer and required improvement of the synthetic techniques (13). An unusual high failure rate in synthesis was noted in southern China, which suggested that environmental factors - such as humidity or temperature - might influence the production procedure or the raw materials.

\section{Additional Diagnostic Gains of Dual-Tracer PET}

In the current study, ${ }^{18} \mathrm{~F}$-FDG and ${ }^{18} \mathrm{~F}-\mathrm{FLT}$ images read in pairs helped in establishing the correct interpretation. The ${ }^{18} \mathrm{~F}$-FLT $/{ }^{18} \mathrm{~F}-\mathrm{FDG}$ ratio was more accurate in revealing the nature of the pulmonary pathologies. A ratio between 0.4 and 0.90 depicted most tumors correctly. A similar finding was recognized when the data of previous studies using both ${ }^{18} \mathrm{~F}$-FDG and ${ }^{18} \mathrm{~F}-\mathrm{FLT}$ were reviewed carefully. Recalculating the assessable data in their studies, we found that the ${ }^{18} \mathrm{~F}-\mathrm{FLT} /{ }^{18} \mathrm{~F}-\mathrm{FDG}$ ratios in 12 of 18 tumors by Yamamoto et al. (3), 8 of 11 tumors by Buck et al. (5), and 8 of 12 tumors by Yap et al. (9) were in the same range. However, in the study of Cobben et al. (14), the ${ }^{18} \mathrm{~F}-$ $\mathrm{FLT} /{ }^{18} \mathrm{~F}-\mathrm{FDG}$ ratio of malignant lesions was lower, but their late ${ }^{18} \mathrm{~F}$-FDG PET imaging at $90 \mathrm{~min}$ after injection might result in higher $\mathrm{SUV}_{\mathrm{FDG}}$ - thus, lowering ${ }^{18} \mathrm{~F}-\mathrm{FLT} /$ ${ }^{18} \mathrm{~F}$-FDG ratio. The higher ${ }^{18} \mathrm{~F}-\mathrm{FLT} /{ }^{18} \mathrm{~F}-\mathrm{FDG}$ ratio in our inflammation was believed to be due to the lower uptake of ${ }^{18} \mathrm{~F}-\mathrm{FDG}$ by the lesions. Two of 3 benign lesions reported by Yap et al also had ${ }^{18} \mathrm{~F}-\mathrm{FLT} /{ }^{18} \mathrm{~F}-\mathrm{FDG}$ ratios $>100 \%$.

There was no clear explanation for the different ${ }^{18} \mathrm{~F}-\mathrm{FLT} /$ ${ }^{18} \mathrm{~F}-\mathrm{FDG}$ ratios among the subgroups of patients. It was known that ${ }^{18} \mathrm{~F}$-FLT is transported into cells via an equilibrative nucleoside transporter-mediated facilitated transport mechanism, phosphorylated by enzyme thymidine kinase 1, and accumulated as mono-, di-, and triphosphate nucleotides in cells (15). It might be correct to assume that all of these biochemical processes keep some sort of balance with the energy metabolism of the cells, resulting in a rather fixed ${ }^{18} \mathrm{~F}$-FLT $/{ }^{18} \mathrm{~F}$-FDG ratio. The difference in ${ }^{18} \mathrm{~F}$-FLT $/{ }^{18} \mathrm{~F}$-FDG SUV ratios between tumors, TB, and inflammation could be useful clinically in separating the different pathologies when neither ${ }^{18} \mathrm{~F}$-FDG nor ${ }^{18} \mathrm{~F}$-FLT could face the challenge alone.

Multimodality imaging has been a hot topic in recent years. Early in 2007, Kim et al. reported that the combination of ${ }^{18} \mathrm{~F}-\mathrm{FDG}$ PET and CT improved the diagnostic efficacy in solitary pulmonary lesions, with the best performance in ROC analysis (16). Another recent report on liver cancer confirmed that dual-tracer PET/CT had an incremental value and a complementary advantage when compared with single- tracer imaging in the evaluation of metastasis (17). In our study, the combination of biologic probes $\left({ }^{18} \mathrm{~F}-\mathrm{FDG}\right.$ and ${ }^{18} \mathrm{~F}-\mathrm{FLT}$ ) and of metabolic and anatomic imaging modalities(PET and CT) increased the diagnostic sensitivity and accuracy in a rather heterogeneous group of patients. Two imaging modalities are definitely better than one.

The radiation burden of dual-tracer, dual-modality imaging of the patients must be addressed. The effective dose equivalent of ${ }^{18} \mathrm{~F}$-FLT was estimated by Vesselle et al. (18) as $0.031 \mathrm{mSv} / \mathrm{MBq}(114 \mathrm{mrem} / \mathrm{mCi})$, which is compatible with that of ${ }^{18} \mathrm{~F}-\mathrm{FDG}(0.029 \mathrm{mSv} / \mathrm{MBq})$. If 2 doses of 400 $\mathrm{MBq}$ were administered in a short interval, the total radiation dose a patient received would be $11.6+12.4=$ $24 \mathrm{mSv}$. In view of the potential benefit that the more confident diagnosis could bring to the patients, the dualtracer strategy should be valued favorably, at least for those with an equivocal diagnosis.

\section{Other Interesting Findings}

SUV and Score. In the current study, a good correlation was found between SUV and score for both ${ }^{18} \mathrm{~F}-\mathrm{FDG}$ and ${ }^{18} \mathrm{~F}$-FLT. It was also shown that the simple scoring based on visual inspection had a diagnostic efficacy similar to that of quantitative SUV. This was in accordance with the reports by Kim et al. (16) and Hashimoto et al. (19) that semiquantitative means, such as SUV, could hardly add diagnostic value over visual inspection in PET.

Dual-Phase Study. Controversial statements about the usefulness and reliability of delayed imaging of ${ }^{18} \mathrm{~F}-\mathrm{FDG}$ have appeared in the literature. In our study, the change in SUV between an early scan and a delayed scan was quite unpredictable. It seemed very unlikely to us that the nature of pulmonary lesions could be differentiated satisfactorily on the basis of dual-phase ${ }^{18} \mathrm{~F}-\mathrm{FDG}$ or ${ }^{18} \mathrm{~F}$-FLT PET.

Value of Low-Dose CT (LDCT). The value of LDCT was appreciated in assisting smaller lesion detection and drawing of ROIs on PET images, especially for ${ }^{18} \mathrm{~F}$-FLT PET. LDCT might miss a few lesions, as $\mathrm{O}$ et al (20) noted, and the unsatisfactory performance of CT in our study might result from the inclusion criteria that precluded the lesion with typical morphologic features.

Limitations of the Trial. There were several limitations in the design and execution of the current MCCT. First, we used LDCT only; no other classical procedures, such as multiplanar reconstruction or contrast enhancement, were undertaken. This might underestimate the value of CT, although the usefulness of LDCT had been verified previously (21). Second, the imaging was acquired and processed using just one model of scanner; the quantitative criteria derived from our study must be cautiously referenced when other devices are used. Third, the number of patients was inadequate, and the composition of diseases was heterogeneous; therefore, not all conclusions may be suitable for the situation in another country or in another study. Because of the limited number of subjects, it was premature to analyze the imaging characteristics according to different histologic 
types of tumors or diseases. Fourth, the 1-y follow-up was not long enough. Thus far, we have no data relating to the influence of dual-tracer PET/CT on the long-term clinical outcome of our patients and we have no evidence on the cost-effectiveness of dual-tracer PET/CT. Last, but not the least, the collective blinded reading was organized with readers of different professional backgrounds and inadequate experience and training, and the adjustment of viewing angle and window settings might influence other readers' attention and judgment-therefore, a subjective factor could not be completely excluded.

\section{CONCLUSION}

In this prospective, randomized multicenter clinical trial, dual-tracer PET/CT using ${ }^{18} \mathrm{~F}-\mathrm{FLT}$ and ${ }^{18} \mathrm{~F}-\mathrm{FDG}$ improved the diagnostic accuracy of differentiating pulmonary nodules. ${ }^{18} \mathrm{~F}-\mathrm{FLT}$ and ${ }^{18} \mathrm{~F}$-FDG reflected different aspects of biologic features but neither tracer alone could guarantee satisfactory diagnostic performance. Although visual inspection scores worked well as quantitative SUVs for both tracers, the SUV ratio between ${ }^{18} \mathrm{~F}$-FLT and ${ }^{18} \mathrm{~F}-\mathrm{FDG}$ worked best in the differentiation of malignancies, TB, and other benign lesions. Considering the limited number of patients and the heterogeneity of underlining diseases of our study population, the real clinical values, long-term clinical impact, and costeffectiveness of the dual-tracer PET/CT in the diagnosis of pulmonary nodules are worthy of further investigation.

\section{ACKNOWLEDGMENTS}

This trial was technically supported and sponsored by GE Healthcare, China, and The Chinese Society of Nuclear Medicine. The contributions of Drs. Hongli Li and Shuang Wang of GE, China, and all staff in the imaging centers are deeply appreciated.

\section{REFERENCES}

1. Strauss LG. Fluoro-18 deoxyglucose and false-positive results: a major problem in the diagnostics of oncological patients. Eur J Nucl Med. 1996;23: 1409-1415.
2. van Waarde A, Cobben DC, Suurmeijer AJ, et al. Selectivity of ${ }^{18} \mathrm{~F}-\mathrm{FLT}$ and ${ }^{18} \mathrm{~F}-$ FDG for differentiating tumor from inflammation in a rodent model. $\mathrm{J} \mathrm{Nucl} \mathrm{Med}$. 2004;45:695-700.

3. Yamamoto Y, Nishiyama Y, Ishikawa S, et al. Correlation of ${ }^{18} \mathrm{~F}$-FLT and ${ }^{18} \mathrm{~F}$ FDG uptake on PET with Ki-67 immunohistochemistry in non-small cell lung cancer. Eur J Nucl Med Mol Imaging. 2007;34:1610-1616.

4. Vesselle H, Grierson J, Muzi M, et al. In vivo validation of 3'-deoxy-3'$\left[{ }^{18} \mathrm{~F}\right]$ fluorothymidine $\left(\left[{ }^{18} \mathrm{~F}\right] \mathrm{FLT}\right)$ as a proliferation imaging tracer in humans: correlation of $\left[{ }^{18} \mathrm{~F}\right] \mathrm{FLT}$ uptake by positron emission tomography with Ki-67 immunohistochemistry and flow cytometry in human lung tumors. Clin Cancer Res. 2002;8:3315-3323.

5. Buck AK, Halter G, Schirrmeister H, et al. Imaging proliferation in lung tumors with PET: ${ }^{18}$ F-FLT versus ${ }^{18}$ F-FDG. J Nucl Med. 2003;44:1426-1431.

6. Bunyaviroch T, Coleman RE. PET evaluation of lung cancer. J Nucl Med. 2006; 47:451-469.

7. Shields AF, Grierson JR, Dohmen BM, et al. Imaging proliferation in vivo with [F-18]FLT and positron emission tomography. Nat Med. 1998;4:1334-1336.

8. Grierson JR, Shields AF. Radiosynthesis of $3^{\prime}$-deoxy- $3^{\prime}-\left[{ }^{18} \mathrm{~F}\right]$ fluorothymidine: $\left[{ }^{18} \mathrm{~F}\right] \mathrm{FLT}$ for imaging of cellular proliferation in vivo. Nucl Med Biol. 2000;27:143-156.

9. Yap CS, Czernin J, Fishbein MC, et al. Evaluation of thoracic tumors with ${ }^{18} \mathrm{~F}-$ fluorothymidine and ${ }^{18} \mathrm{~F}$-fluorodeoxyglucose-positron emission tomography. Chest. 2006;129:393-401.

10. Reske SN, Deisenhofer S. Is $3^{\prime}$-deoxy- $3^{\prime}-{ }^{18}$ F-fluorothymidine a better marker for tumor response than ${ }^{18} \mathrm{~F}$-fluorodeoxyglucose? Eur J Nucl Med Mol Imaging, 2006;33(suppl 1):38-43.

11. Hinrich A, Wieder HA, Geinitz $\mathrm{H}$, et al. PET imaging with $\left[{ }^{18} \mathrm{~F}\right] 3^{\prime}$-deoxy- $3^{\prime}-$ fluorothymidine for prediction of response to neoadjuvant treatment in patients with rectal cancer. Eur J Nucl Med Mol Imaging. 2007;34:878-883.

12. Glaser M, Luthro S, Brady F. Applications of positron-emitting halogens in PET oncology. Int J Oncol. 2003;22:253-267.

13. Oh SJ, Mosdzianowski C, Chi DY, et al. Fully automated synthesis system of $3^{\prime}-$ deoxy-3'-[18F]fluorothymidine. Nucl Med Biol. 2004;31:803-809.

14. Cobben DC, Elsinga PH, Hoekstra HJ, et al. Is ${ }^{18} \mathrm{~F}-3^{\prime}$-fluoro- $3^{\prime}$-deoxy-Lthymidine useful for the staging and restaging of non-small cell lung cancer? J Nucl Med. 2004;45:1677-1682.

15. Grierson JR, Schwartz JL, Muzi M, et al. Metabolism of 3'-deoxy-3'-[F18]fluorothymidine in proliferating A549 cells: validations for positron emission tomography. Nucl Med Biol. 2004;31:829-837.

16. Kim SK, Allen-Auerbach M, Goldin J, et al. Accuracy of PET/CT in characterization of solitary pulmonary lesions. J Nucl Med. 2007;48:214-220.

17. Ho CL, Chen SR, Yeung DWC, et al. Dual-tracer PET/CT imaging in evaluation of metastatic hepatocellular carcinoma. J Nucl Med. 2007;48:902-909.

18. Vesselle H, Grierson J, Peterson LM, Muzi M, Mankoff DA, Krohn KA. ${ }^{18} \mathrm{~F}-$ Fluorothymidine radiation dosimetry in human PET imaging studies. $\mathrm{J} \mathrm{Nucl}$ Med. 2003;44:1482-1488.

19. Hashimoto Y, Tsujikawa T, Kondo C, et al. Accuracy of PET for diagnosis of solid pulmonary lesions with ${ }^{18} \mathrm{~F}-\mathrm{FDG}$ uptake below the standardized uptake value of 2.5. J Nucl Med. 2006;47:426-431.

20. O JH, Yoo IR, Kim SH, et al. Clinical significance of small pulmonary nodules with little or no ${ }^{18} \mathrm{~F}-\mathrm{FDG}$ uptake on PET/CT images of patients with nonthoracic malignancies. J Nucl Med. 2007;48:15-21.

21. Schöder H, Yeung HWD, Larson SM. CT in PET/CT: essential features of interpretation. J Nucl Med. 2005;46:1249-1251. 